\title{
Estudio de modelos matemáticos de Líneas de transmisión para simulación de transitorios electromagnéticos en sistemas de potencia
}

\section{Mathematical models of transmission lines for electromagnetic transient simulations in power systems}

\author{
Jaimis Leon', Sérgio Kurokawa ${ }^{1}$ \\ ${ }^{1}$ Facultad de Ingeniería Eléctrica, Universidad Estadual Paulista "Júlio de Mesquita Filho", UNESP, Ilha Solteira-Brasil.
} Recibido (Received): 14/10/2018 Aceptado (Accepted): 05/12/2018

\section{RESUMEN}

En este trabajo se estudian tres modelos matemáticos de líneas de transmisión, Cascada de Circuito PI, Bergeron y Universal Line Model para simular transitorios electromagnéticos en sistemas de potencia. Se exponen sus principales características, ventajas y desventajas. Asimismo, se muestran los algoritmos en forma de flujogramas para las simulaciones de los transitorios. Finalmente, se presentan los resultados de simulaciones de sobretensiones de maniobra en una línea de transmisión monofásica utilizando el software MATLAB. Se comprueba que el Universal Line Model es el más preciso.

Palabras Clave: Modelos de Líneas de transmisión, transitorios electromagnéticos, dominio del tiempo, modelo matemático.

\section{ABSTRACT}

In this work we study three mathematical models of transmission lines, Cascade of PI Circuit, Bergeron and Universal Line Model to simulate electromagnetic transients in power systems. its main features, advantages and disadvantages are discussed. Likewise, the algorithms are shown in the form of flow charts for the transient simulations. Finally, the results of simulations of maneuvering surges in a single-phase transmission line using the MATLAB software are presented. It is verified that the Universal Line Model is the most accurate.

Keywords: Transmission line models, electromagnetic transient, time domain, mathematical model.

\section{INTRODUCCIÓN}

En los sistemas eléctricos de potencia, y en general en las líneas de transmisión (LT), se presentan fenómenos transitorios tales como: sobretensiones temporales, sobretensiones por maniobra y sobretensiones por descargas atmosféricas [1]. Para entender el comportamiento de estos fenómenos, necesitamos modelos de LT que representan con exactitud estos transitorios electromagnéticos (TEM).

Los modelos de las LT varían en complejidad y eficiencia, dependiendo de la precisión requerida. Los parámetros que constituyen estos modelos pueden ser constantes o dependientes de la frecuencia, también pueden ser clasificados como parámetros de la LT concentrados o distribuidos.

\footnotetext{
* Correspondencia:

E-mail: jaimis.leon@unesp.br
}

Un modelo clásico para representar la LT, se basa en una cascada de circuitos $\pi$ [1-3]. Este modelo es considerado a parámetros concentrados y constantes. Sin embargo, al hacer esta cascada se produce la naturaleza distribuida de los parámetros de la LT.

Este modelo es utilizado en simulaciones de sobretensiones de maniobra y conmutación. Para análisis de TEM, este modelo es usado para LT de interés secundario, debido a que presentan en sus respuestas oscilaciones numéricas.

Otro modelo de LT es el modelo de Bergeron, que se basa en la teoría de ondas viajantes, por lo que resulta un modelo simple y eficiente $[1,4]$. Este modelo está constituido por parámetros constantes y distribuidos. Una desventaja de este modelo es la no dependencia con la frecuencia de los parámetros longitudinales, pero puede ser utilizado para representar la LT tanto 
en simulaciones de sobretensiones por maniobra, conmutación y descargas atmosféricas.

Por último, el modelo capaz de representar con precisión los TEM de las LT es el Universal Line Model (ULM) [5]. Este modelo está constituido por parámetros de la LT distribuidos y dependientes de la frecuencia, y tiene como base la teoría de ondas viajantes.

El objetivo del trabajo es determinar cuál de los tres modelos de LT, para simular TEM, es más preciso. En este sentido son mostrados sus modelos matemáticos y algoritmos que tienen como base flujogramas para las simulaciones. También, es mostrado resultados de sobretensiones de maniobra en una LT monofásica. Se formula como hipótesis que el ULM es el modelo más preciso para simular transitorios en una LT. La clasificación de los modelos se ilustra en la Figura 1.

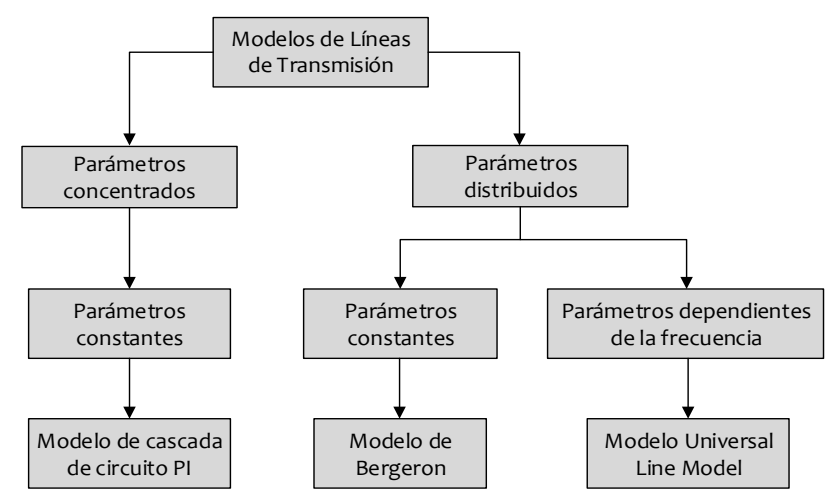

Figura 1. Clasificación de los diferentes modelos de LT [1].

\section{MODELO DE CASCADA DE CIRCUITOS $\pi$ (MODELO A PARÁMETROS CONCENTRADOS CONSTANTES)}

El modelo de LT a parámetros concentrados es empleado para simulaciones transitorias de energización y conmutación. Este modelo de LT puede ser representada por una cascada de circuitos $\pi[1,2]$. Cada segmento de circuito $\pi$ consta de una resistencia y una inductancia en serie. Completando el circuito $\pi$, hay componentes en paralelo: capacitancia $y$ conductancia. En la Figura 2 se muestra un elemento de circuito $\pi[2,3]$.

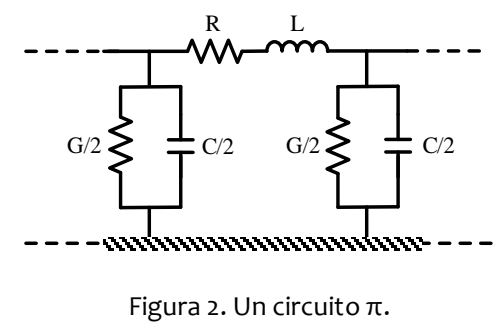

La LT monofásica de longitud d, representada por la cascada de circuitos $\pi$, se muestra en la Figura 3 [2].

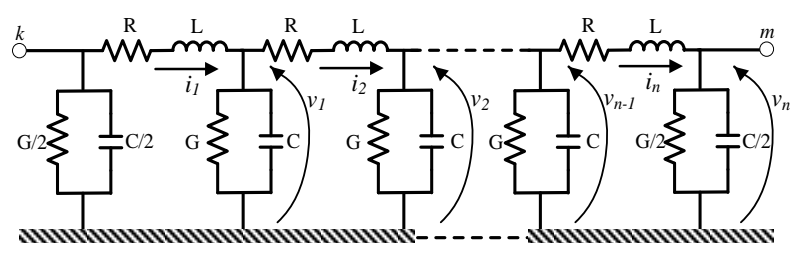

Figura 3. LT representada por una cascada de circuitos $\pi$.

En la Figura 3, los parámetros $R$ y $L$ son la resistencia e inductancia de cada circuito $\pi$ respectivamente. Los parámetros $G$ y $C$ son la conductancia y la capacitancia de cada circuito $\pi$ respectivamente. Los parámetros $R, L, G$ y $C$ son escritos como:

$$
R=R^{\prime} \frac{d}{n}, L=L^{\prime} \frac{d}{n}, C=C^{\prime} \frac{d}{n}, G=G^{\prime} \frac{d}{n}
$$

En la ecuación (1) los parámetros longitudinales R', L' y parámetros trasversales C'y G' son datos por unidad de longitud y $n$ es el número de circuitos $\pi$ usados en la representación de la LT. En base a los circuitos $\pi$ de la Figura 3 es posible determinar las siguientes ecuaciones diferenciales [2]:

$$
\begin{gathered}
\frac{d i_{1}}{d t}=\dot{i}_{1}=\frac{1}{L}\left(u-R \cdot i_{1}-v_{1}\right) \\
\frac{d v_{1}}{d t}=\dot{v}_{1}=\frac{1}{C}\left(i_{1}-G \cdot v_{1}-i_{2}\right)
\end{gathered}
$$

De las ecuaciones (2) y (3), se nota la influencia de la tensión y corriente eléctrica del circuito $\pi$ anterior y posterior, respectivamente, en el circuito $\pi$ especifico. Si definimos las corrientes en los inductores y las tensiones en los capacitores como variables de estado, el sistema de ecuaciones diferenciales resultantes para la cascada de circuitos $\pi$ de la Figura 3 puede ser escrito como $[2,3]$ :

$$
\frac{d[x]}{d t}=A[x]+B[u]
$$

En la ecuación (4), A y B son matrices de estado para la $L T,[x]$ es el vector de variables de estado y $[u]$ es el vector de entrada de datos. Estas matrices y vectores son definidos a continuación [2]: 


$$
\begin{gathered}
A=\left[\begin{array}{ccccccc}
\frac{1}{L} & -\frac{R}{L} & -\frac{1}{L} & 0 & \cdots & \cdots & 0 \\
0 & \frac{1}{C} & -\frac{G}{C} & -\frac{1}{C} & \ddots & \ddots & \vdots \\
\vdots & \ddots & \ddots & \ddots & \ddots & \ddots & \vdots \\
\vdots & \ddots & \ddots & \frac{1}{C} & -\frac{G}{C} & -\frac{1}{C} & 0 \\
\vdots & \ddots & \ddots & \ddots & \frac{1}{L} & -\frac{R}{L} & -\frac{1}{L} \\
0 & \cdots & \cdots & \cdots & 0 & \frac{2}{C} & -\frac{G}{C}
\end{array}\right] \\
{[x]=\left[\begin{array}{llllllllll}
i_{1} & v_{1} & i_{2} & v_{2} & \cdots & i_{j} & v_{j} & \cdots & i_{n} & v_{n}
\end{array}\right]^{\mathrm{T}}}
\end{gathered}
$$

En las ecuaciones (5), (6) y (7), la matriz $A$ es cuadrada de orden $2 n$, la matriz $B$ y matriz $[x]$ tienen orden 2 nx1.

El sistema de ecuaciones en (4) puede ser solucionado por diversos métodos numéricos que resuelven ecuaciones diferenciales. En [2] se evaluaron diferentes métodos de integración, siendo la regla de integración trapezoidal el método más robusto, ya que la precisión de este se mosto menos sensible en función del aumento del paso de cálculo.

La ecuación (8) es resultado de usar el método de integración trapezoidal $[2,3]$.

$$
[x]_{j+1}=[x]_{j}+\frac{\Delta t}{2}\left(A[x]_{j}+B[u]_{j}+A[x]_{j+1}+B[u]_{j+1}\right)
$$

Simplificando la ecuación (8), obtenemos [2,3]:

$$
[x]_{j+1}=A^{\prime \prime}[x]_{j}+B "\left([u]_{j}+[u]_{j+1}\right)
$$

Donde A', A" y B son matrices constantes que son descritas en las ecuaciones (10), (11) y (12).

$$
\begin{gathered}
A^{\prime}=\left[I-\frac{T}{2} A\right]^{-1} \\
A^{\prime \prime}=A^{\prime}\left[I+\frac{T}{2} A\right] \\
B^{\prime}=A^{\prime} \frac{T}{2} B
\end{gathered}
$$

El algoritmo de este modelo utilizado para realizar las simulaciones de los TEM tiene como base el flujograma que se muestra en la Figura 4.

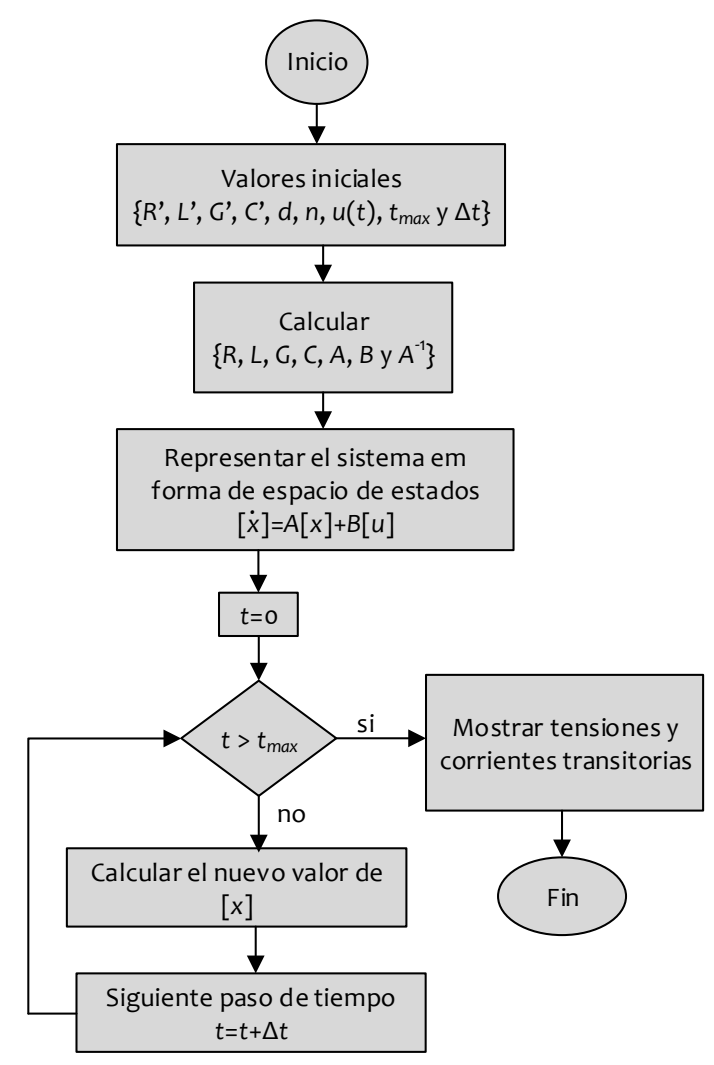

Figura 4. Algoritmo del modelo de cascada de circuitos $\pi$.

\section{MODELO DE BERGERON (MODELO A PARÁMETROS DISTRIBUIDOS CONSTANTES)}

El modelo de Bergeron [1,4] es un método de frecuencia constante basado en la teoría de las ondas viajantes. Inicialmente este modelo era utilizado para líneas de transmisión sin perdidas, pero después en [4] una extensión del modelo fue desarrollado para incluir las pérdidas de la LT. La inclusión de las pérdidas fue obtenida agregando elementos de resistencia concentradas en ambos extremos de la LT sin perdida. Aunque estas resistencias se pueden insertar a lo largo de la línea dividiendo su longitud total en varias secciones, es mínima la diferencia y el uso de apenas dos secciones en serie con las resistencias concentradas es perfectamente adecuado. En la Figura 5 se muestra este modelo aproximado de la LT [4].

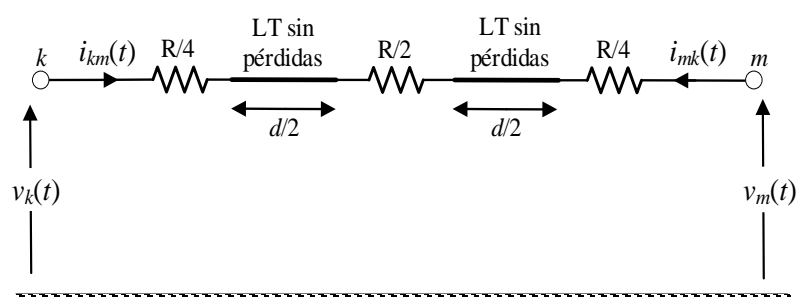

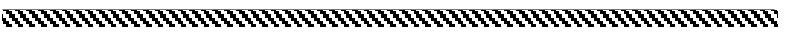

Figura 5. Modelo de la LT con pérdidas.

Este modelo, proporciona respuestas razonables, siempre que el valor de las resistencias concentradas sea mucho menor al valor de la impedancia 
característica de la línea [4]. Sin embrago, para estudios de alta frecuencia (por ejemplo, portadora de línea de potencia), este modelo puede no ser adecuado. El circuito equivalente de la Figura 5 en representación Norton se muestra en la Figura 6 [4].

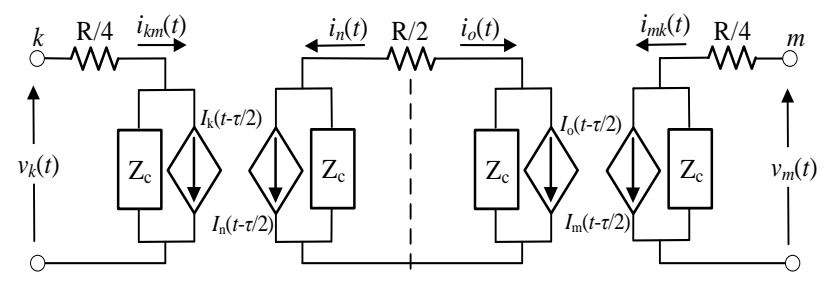

Figura 6. Circuito equivalente de la LT con pérdidas.

De la Figura 6, es obtenido un modelo para cada mitad de LT con pérdidas. Sin embargo, como solo los terminales $\mathrm{k}$ y $\mathrm{m}$ son de interés, son eliminadas las variables del punto medio colocando en cascada las dos secciones de media LT. En consecuencia, se obtiene el modelo de LT que se muestra en la Figura 7 [4].

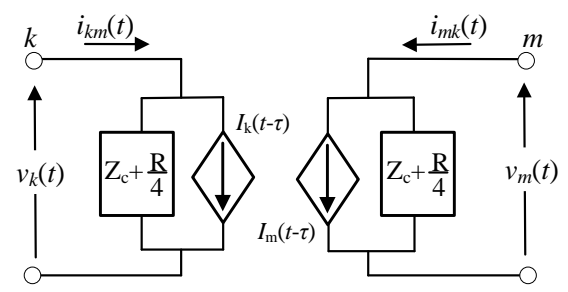

Figura 7. Modelo de Bergeron para LT.

A partir de un análisis realizado en [4], se obtiene las expresiones para las corrientes, en el dominio de tiempo, para los terminales emisor y receptor.

$$
\begin{aligned}
& i_{m k}(t)=\frac{1}{Z} v_{m}(t)+I_{\mathrm{m}}(t-\tau) \\
& i_{k m}(t)=\frac{1}{Z} v_{k}(t)+I_{k}(t-\tau)
\end{aligned}
$$

De las ecuaciones (13) y (14), se observa que las corrientes $i_{m k}$ e $i_{k m}$ en el instante $t$ se determinan a partir de las corrientes históricas en el tiempo $(t-\tau)$. Estas corrientes históricas son mostradas en las siguientes ecuaciones [4].

$$
\begin{aligned}
& I_{k}(t-\tau)=\left(\frac{1+h}{2}\right)\left[\left(\frac{-1}{Z}\right) v m(t-\tau)-h \cdot i m k(t-\tau)\right] \\
&+\left(\frac{1-h}{2}\right)\left[\left(\frac{-1}{Z}\right) v k(t-\tau)-h \cdot k k m(t-\tau)\right] \\
& I_{m}(t-\tau)=\left(\frac{1+h}{2}\right)\left[\left(\frac{-1}{Z}\right) v k(t-\tau)-h \cdot k k m(t-\tau)\right] \\
&+\left(\frac{1-h}{2}\right)\left[\left(\frac{-1}{Z}\right) v m(t-\tau)-h \cdot i m k(t-\tau)\right]
\end{aligned}
$$

Donde:

$$
\tau=l \sqrt{L C}
$$

$$
\begin{gathered}
Z c=\sqrt{L / C} \\
Z=Z c+R / 4 \\
h=(Z c-R / 4) /(Z c+R / 4)
\end{gathered}
$$

En la Figura 8, es mostrado el algoritmo en forma de flujograma del modelo de Bergeron para realizar simulaciones de los TEM.

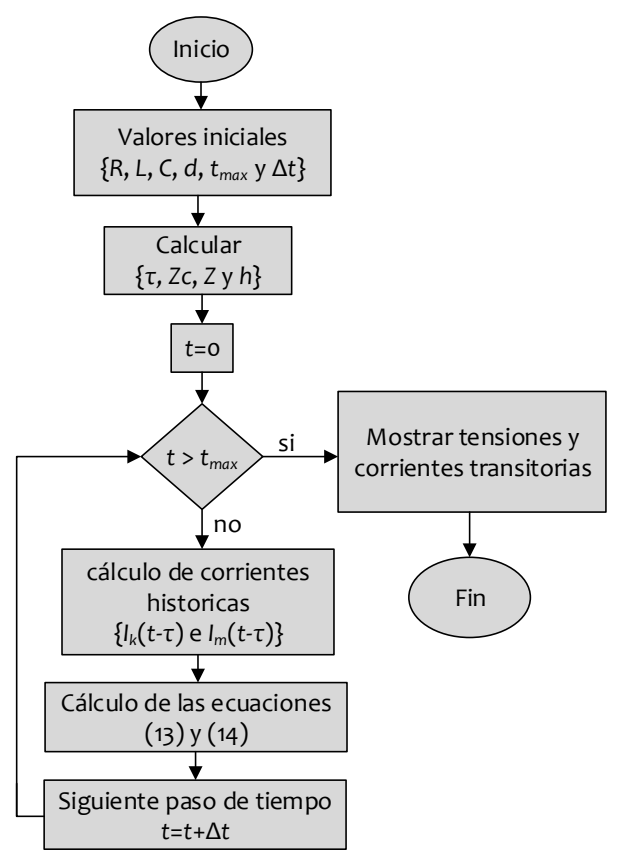

Figura 8. Algoritmo del modelo de Bergeron.

\section{UNIVERSAL LINE MODEL (ULM - MODELO A PARÁMETROS DISTRIBUIDOS E DEPENDIENTES DE LA FRECUENCIA)}

El ULM [5] se basa en la formulación del tipo de onda viajante y en las ecuaciones del Telegrafista, que en el dominio de la frecuencia se expresan de la siguiente manera [5]:

$$
\begin{aligned}
& \frac{d V}{d x}=-Z I \\
& \frac{d I}{d x}=-Y V
\end{aligned}
$$

Donde $V$ e I son las tensiones y corrientes de fase a lo largo del conductor, $Z$ e $Y$ son respectivamente la impedancia en serie y la admitancia shunt, ambos en por unidad de longitud.

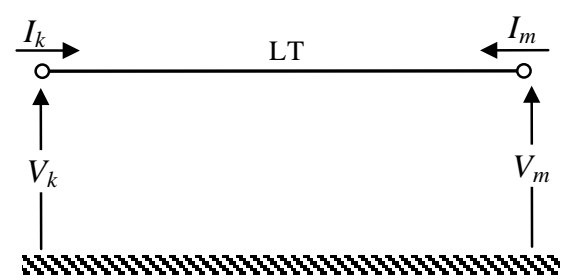

Figura 9. Diagrama de la línea de transmisión monofásica.

Revista TECNIA Vol. 28 N 1 Agosto - Diciembre 2018 
Dado un segmente de línea de transmisión de longitud $d$ como el representado en la Figura 9, la solución de (21) y (22) en términos de corrientes se expresan de la siguiente manera [5]:

$$
\begin{aligned}
& I_{k}=Y_{C} V_{k}-H\left[I_{m}+Y_{C} V_{m}\right] \\
& I_{m}=Y_{C} V_{m}-H\left[I_{k}+Y_{C} V_{k}\right]
\end{aligned}
$$

Los subíndices $k$ y $m$ indican los valores de $V$ y $I$ de los terminales emisor y receptor, respectivamente. $Y_{c} y$ $H$ son respectivamente la admitancia característica y función propagación para las ondas de corriente [5]:

$$
\begin{gathered}
Y_{c}=\sqrt{Y Z} Z^{-1} \\
H=e^{-\sqrt{Y Z} d}
\end{gathered}
$$

En la Figura 10 [5] es mostrada la representación en el dominio de la frecuencia de los terminales de la línea con dos equivalentes Norton. Ambos equivalentes conforman el modelo completo de la línea de transmisión monofásica. Téngase en cuenta que este modelo no tiene conexión directa entre los dos extremos de la línea. Su relación es solo a través de las fuentes de corriente auxiliar que implican un retraso de tiempo. Por lo tanto, se dice que los dos extremos están desacoplados en el tiempo.

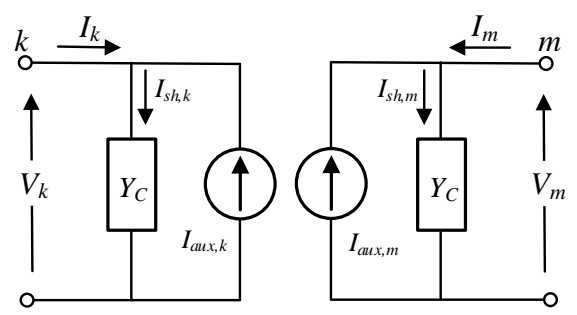

Figura 10. Diagrama de la línea de transmisión monofásica.

Las ecuaciones de la línea descritas en (23) y (24) deben resolverse en el dominio del tiempo para simular transitorios electromagnéticos. Por lo tanto, la forma general en el dominio del tiempo da como resultado las siguientes ecuaciones [5]:

$$
\begin{aligned}
& i_{k}(t)=y_{C}(t) * v_{k}(t)-h(t) *\left[i_{m}(t)+y_{C}(t) * v_{m}(t)\right] \\
& i_{m}(t)=y_{C}(t) * v_{k}(t)-h(t) *\left[i_{k}(t)+y_{C}(t) * v_{k}(t)\right]
\end{aligned}
$$

Donde las variables en minúscula son la representación en el dominio del tiempo de sus contrapartes en mayúsculas y el símbolo * indica una convolución. La solución de las ecuaciones (27) y (28) se llevan a cabo de manera muy eficiente a través del análisis del espacio de estados. Esto se hace cuando $Y_{c}$ y $\mathrm{H}$ se representan como funciones racionales [5].

$$
H=\sum_{p=1}^{N} \frac{c_{p} e^{-s t}}{s-a_{p}}
$$

$$
Y_{c}=\sum_{q=1}^{N} \frac{c_{q}}{s-a_{q}}+f
$$

Los coeficientes desconocidos $c_{p}, a_{p}, a_{q}$ y $f$ se calculan utilizando la técnica llamada Vector Fitting [5] de la representación de $\mathrm{Yc}$ y $\mathrm{H}$.

Una forma compacta de la ecuación (27) es [5]:

$$
i_{k}(t)=i_{s h, k}(t)-i_{a u x, k}(t)
$$

Donde:

$$
\begin{gathered}
i_{s h, k}(t)=y_{c}(t) * v_{k}(t) \\
i_{a u x, k}(t)=h(t) * i_{r f t, m}(t) \\
i_{r f t, m}(t)=i_{m}(t)+y_{C}(t) * v_{m}(t)
\end{gathered}
$$

La misma idea de la forma compacta de la ecuación (27) se aplica para la ecuación (28).

Finalmente, para implementar el modelo ULM en el dominio del tiempo las convoluciones de las ecuaciones (27) y (28), se realizan usando métodos de espacio de estado discreto [5] que son de la siguiente forma:

$$
\dot{x}(t)=A x(t)+B v(t)
$$

En la Figura 11, se muestra el algoritmo en forma de flujograma del ULM para realizar simulaciones de los TEM.

\section{SIMULACIÓN DE TRANSITORIOS Y ANÁLISIS DE RESULTADOS}

Las simulaciones fueron realizadas para una LT monofásica de $100 \mathrm{~km}$ de longitud, siendo energizada por dos tipos de fuentes: sinusoidal y escalón unitario, en el terminal emisor. Por otro lado, el terminal receptor se encuentra en dos estados: circuito abierto y conectado a una carga. Para el cálculo de las impedancias por unidad de longitud, la LT fue considerada de acuerdo a la Figura 12. En los tres modelos de la LT son calculadas las tensiones en el terminal receptor. La implementación de algoritmo de los modelos fue desarrollado en el lenguaje de programación Matlab. 


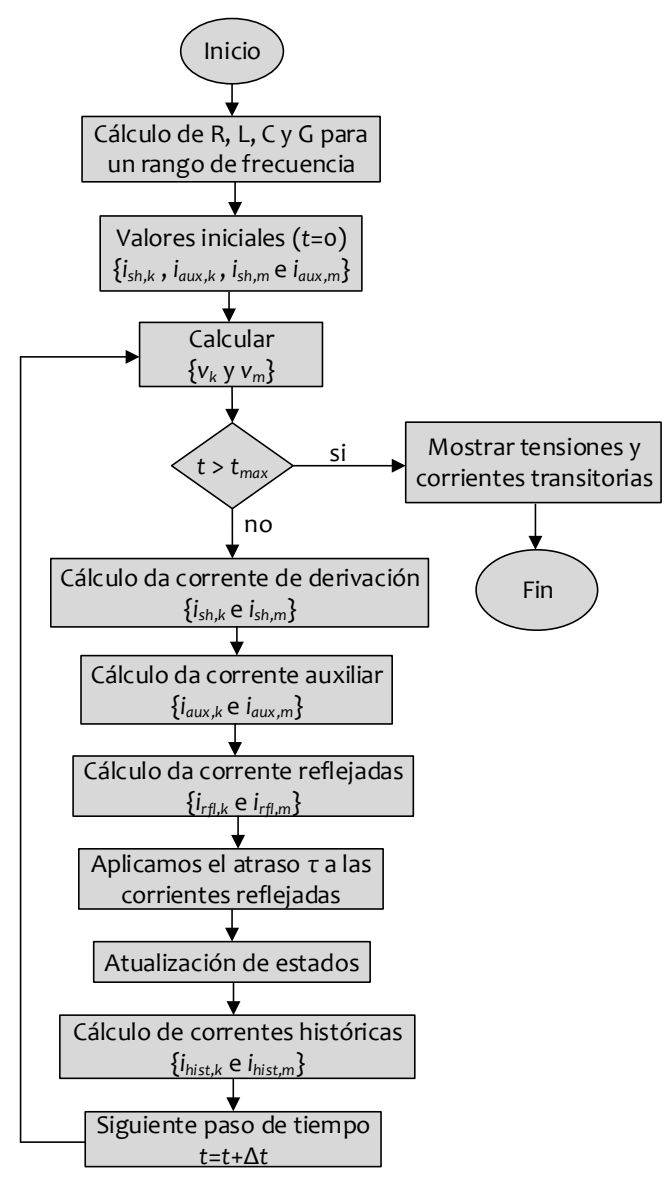

Figura 11. Algoritmo del ULM.

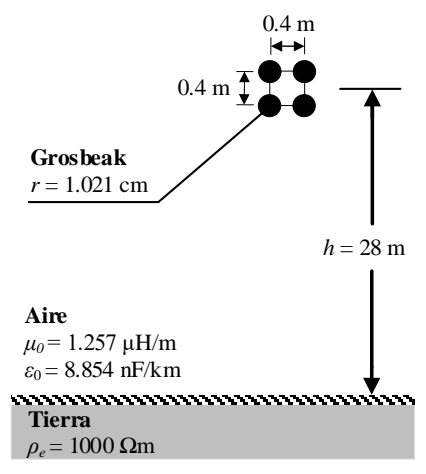

Figura 12. LT aérea.

Para todas las simulaciones, se tuvo que $V_{0}=100$ kV y $\omega=377 \mathrm{rad} / \mathrm{s}$ y los resultados fueron presentados en valores por unidad (p.u.) siendo $100 \mathrm{kV}$ el valor base. Los parámetros longitudinales (resistencia e inductancia) fueron calculadas para un ancho de frecuencia de $0.1 \mathrm{~Hz}$ a $1 \mathrm{MHz}$ y sus comportamientos son mostrados en la Figura 13. En las Figuras $14-17$ se muestran los transitorios de las tensiones en el terminal receptor obtenidas con los modelos cascada de circuitos $\pi$ (LPM), Bergeron (MB) y Universal Line Model (ULM).

Para el modelo LPM se utilizaron 100 circuitos $\pi$ y los parámetros constantes fueron calculados a $60 \mathrm{~Hz}$, observándose la presencia de oscilaciones numéricas que no representan el valor real de las simulaciones y que pueden ser mejoradas a través de filtros o usando DOI: https://doi.org/10.21754/tecnia.v28i2.550 circuitos $\pi$ modificados. Para el modelo MB, los parámetros usados fueron calculados, también, a 60 Hz. Para el modelo ULM, los parámetros usados fueron calculados para un ancho de frecuencia de $0.1 \mathrm{~Hz}$ a 1 $\mathrm{MHz}$.

En las Figuras 14 y 16 se observa que la tensión se duplica en la primera reflexión. En las Figuras 15 y 17 se observa que, al conectar una carga $(5 \mathrm{k} \Omega$ ) en el terminal receptor, las sobretensiones se atenúan debido a que la resistencia las amortigua.
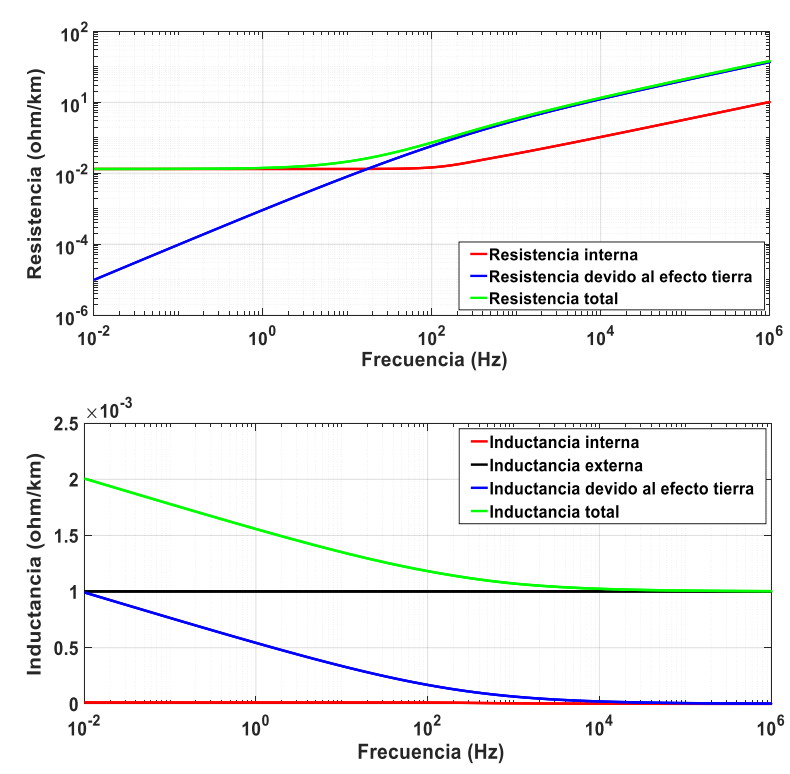

Figura 13. Parámetros longitudinales de LT.

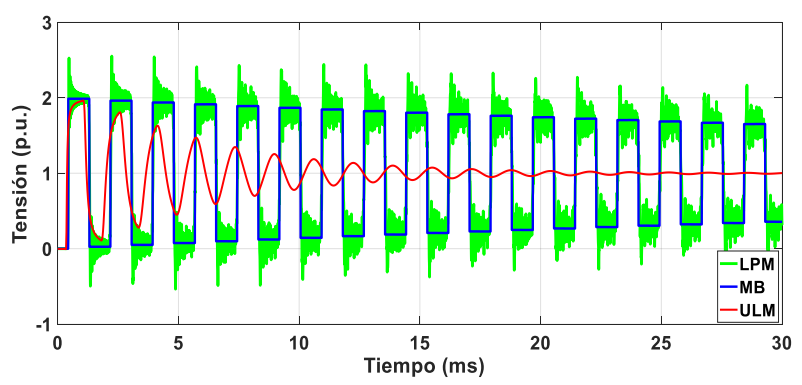

Figura 14. Energización de LT en circuito abierto y fuente escalón.

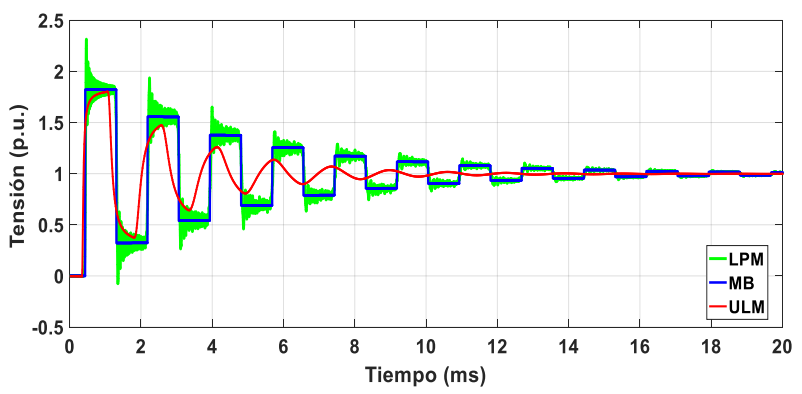

Figura 15. Energización de LT con carga de $5 \mathrm{k} \Omega$ y fuente escalón.

Revista TECNIA Vol. 28 N 1 Agosto - Diciembre 2018 


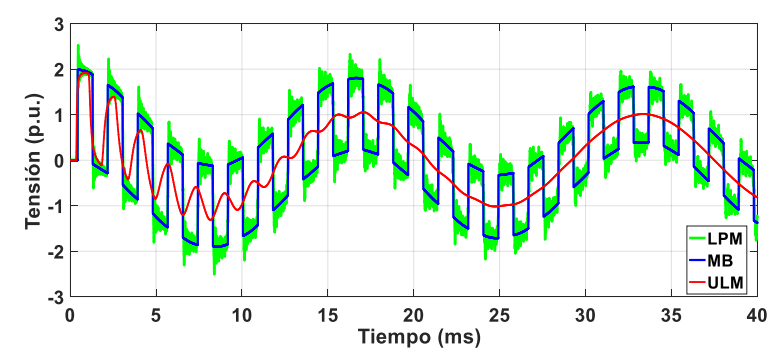

Figura 16. Energización de LT en circuito abierto y fuente senoidal.

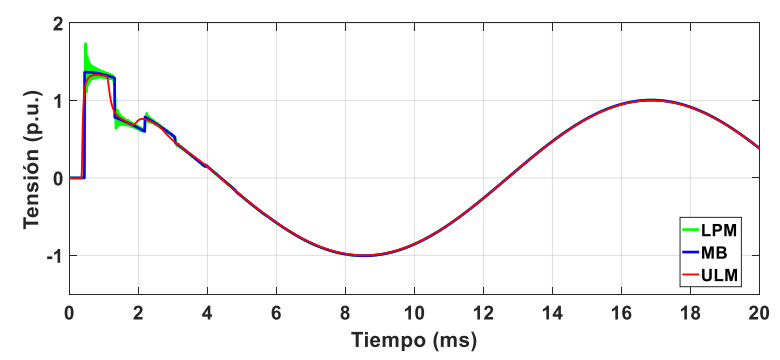

Figura 17. Energización de LT con carga de $5 \mathrm{k} \Omega$ y fuente senoidal.

\section{CONCLUSIONES}

En este trabajo se describieron tres modelos matemáticos de líneas de transmisión para el cálculo de transitorios electromagnéticos, demostrándose que el modelo Universal line Model es el más preciso; validándose así la hipótesis del trabajo.

Se comprobó la utilidad práctica de los algoritmos en forma de flujograma que se desarrollaron para cada modelo ya que con ellos se realizaron las simulaciones de transitorios electromagnéticos en el modelo monofásico de una línea de transmisión energizada.

\section{AGRADECIMIENTOS}

Agradezco a la Facultad de Ingeniería Eléctrica de la UNESP-Brasil por el soporte académico. También agradecer al Consejo Nacional de Desarrollo Tecnológico y Científico CNPq-Brasil por el soporte económico dado para poder realizar este trabajo.

\section{REFERENCIAS}

[1] Restrepo L., Caicedo, G., Castro. F. (2008). Modelos de línea de transmisión para transitorios electromagnéticos en sistemas de potencia. Revista Energía y Computación, v. 16, p. 21-32.

[2] Araujo A., Silva R. and Kurokawa S. (2014). Comparing Lumped and Distributed Parameters Models in Transmission Lines during Transient Conditions. IEEE PES T\&D Conference and Exposition.

[3] Dommel, H. (1969). Digital computer solution of electromagnetic transients in single and multiphase networks. IEEE Transactions on Power Apparatus and Systems, v. 88, p. 388-399.

[4] Mamis M., Kaygusuz A. and Koksal M. (2010). State variable distributed-parameter representation of transmission line for transient simulation. Turkish J Elec Eng \& Comp Sci, v. 18, p. 31-42.

[5] Ramos-Leaños O., Naredo J. and Gutierrez J. (2012). An Advanced Transmission Line and Cable Model in Matlab for the Simulation of Power System Transients in MATLAB. InTech Open A Fundamental Tool for Scientific Computing and Engineering Applications. v. 1, p. 269-304.
Los artículos publicados por TECNIA pueden ser compartidos a través de la licencia Creative Commons: CC BY 4.o Perú. Permisos lejos de este alcance pueden ser consultados a través del correorevistas@uni.edu.pe 